\title{
STATISTICAL APPROACH FOR ASSESSING THE STOCKHOLM CONVENTION'S EFFECTIVENESS: GREAT LAKES ATMOSPHERIC DATA
}

\author{
Ronald A. Hites* \\ O’Neill School of Public and Environmental Affairs \\ Indiana University \\ Bloomington, IN 47405
}

Table S1. Dates (in month/day/year) for the first samples used in this analysis. Ending dates were all 12/31/2016. The number of samples at each site $(N)$ for each compound is given in Table S2. NM: indicates that this compound was not measured.

\begin{tabular}{|c|c|c|c|c|c|c|}
\hline \multicolumn{7}{|c|}{ Vapor Phase } \\
\hline & Chicago & Cleveland & Sturgeon Point & Sleeping Beard Dunes & Point Petre & Eagle Harbor \\
\hline Chlordanes & $1 / 11 / 1996$ & $1 / 9 / 2003$ & $8 / 19 / 1992$ & $8 / 7 / 1992$ & $1 / 6 / 1999$ & $7 / 26 / 1992$ \\
\hline DDTs & $1 / 11 / 1996$ & $1 / 9 / 2003$ & 1/16/1992 & 1/1/1992 & 1/6/1999 & 1/6/1992 \\
\hline Endosulfans & $1 / 11 / 1996$ & $1 / 9 / 2003$ & $11 / 16 / 1994$ & $12 / 10 / 1994$ & $1 / 6 / 1999$ & $11 / 16 / 1994$ \\
\hline$\alpha-\mathrm{HCH}$ & $1 / 11 / 1996$ & $1 / 9 / 2003$ & $1 / 16 / 1992$ & $1 / 1 / 1992$ & $1 / 6 / 1999$ & $1 / 16 / 1992$ \\
\hline$\gamma-\mathrm{HCH}$ & $1 / 11 / 1996$ & $1 / 9 / 2003$ & $1 / 16 / 1992$ & $1 / 1 / 1992$ & $1 / 6 / 1999$ & $1 / 16 / 1992$ \\
\hline PCB-18 & $1 / 11 / 1966$ & $1 / 9 / 2003$ & $1 / 16 / 1992$ & $1 / 1 / 1992$ & $5 / 23 / 1998$ & $1 / 16 / 1992$ \\
\hline PCB-52 & $1 / 11 / 1996$ & $1 / 9 / 2003$ & $1 / 16 / 1992$ & $1 / 1 / 1992$ & $5 / 23 / 1998$ & $1 / 16 / 1992$ \\
\hline PCB-101 & $1 / 11 / 1996$ & $1 / 9 / 2003$ & $1 / 16 / 1992$ & $1 / 1 / 1992$ & $5 / 23 / 1998$ & $1 / 16 / 1992$ \\
\hline PCB total & $1 / 11 / 1996$ & $1 / 9 / 2003$ & $1 / 16 / 1992$ & 1/1/1992 & $5 / 23 / 1998$ & $1 / 16 / 1992$ \\
\hline PAH total & $1 / 11 / 1996$ & $1 / 9 / 2003$ & $1 / 16 / 1992$ & $1 / 1 / 1992$ & $5 / 23 / 1998$ & $1 / 16 / 1992$ \\
\hline Phenanthrene & $1 / 11 / 1996$ & $1 / 9 / 2003$ & $1 / 16 / 1992$ & $1 / 1 / 1992$ & $5 / 23 / 1998$ & $1 / 16 / 1992$ \\
\hline \multicolumn{7}{|c|}{ Particle Phase } \\
\hline & Chicago & Cleveland & Sturgeon Point & Sleeping Bear Dunes & Point Petre & Eagle Harbor \\
\hline Chlordanes & $10 / 13 / 1996$ & $1 / 9 / 2003$ & NM & NM & NM & NM \\
\hline DDTs & $10 / 13 / 1996$ & $1 / 9 / 2003$ & NM & NM & NM & NM \\
\hline Endosulfans & $10 / 13 / 1996$ & $1 / 9 / 2003$ & NM & NM & NM & NM \\
\hline PAH total & $10 / 13 / 1996$ & $1 / 9 / 2003$ & $10 / 1 / 1996$ & $10 / 1 / 1996$ & $5 / 23 / 1998$ & $10 / 1 / 1996$ \\
\hline Phenanthrene & $10 / 13 / 1996$ & $1 / 9 / 2003$ & $10 / 1 / 1996$ & $10 / 1 / 1996$ & $5 / 23 / 1998$ & $10 / 1 / 1996$ \\
\hline
\end{tabular}


Table S2. Detailed regression results from eqs. 4 and 6 for 11 compounds in the vapor (top) and particle (bottom) phases. The errors are all standard errors. The blue font indicates that the parameter was not statistically significant.

\begin{tabular}{|c|c|c|c|c|c|c|c|c|c|c|c|c|c|c|c|c|c|c|c|c|c|c|}
\hline \multirow{2}{*}{$\begin{array}{l}\text { Compound } \\
\text { Chlordanes }\end{array}$} & \multirow{2}{*}{$\begin{array}{l}\text { phase } \\
\text { vapor }\end{array}$} & \multirow{2}{*}{$\begin{array}{c}\text { t_avg } \\
12 / 7 / 2005\end{array}$} & \multirow{2}{*}{$\underset{3274}{\mathbf{N}}$} & \multirow{2}{*}{$\begin{array}{c}\mathbf{r}^{2} \\
0.570\end{array}$} & \multicolumn{2}{|c|}{ a_0 (intcp) } & \multicolumn{2}{|c|}{ a_1 (t) } & \multicolumn{2}{|c|}{ a_2 (sin) } & \multicolumn{2}{|c|}{ a_3 (cos) } & \multicolumn{2}{|c|}{ a_4 (pop) } & \multicolumn{2}{|c|}{ a_5 $\left(t^{2}\right)$} & \multicolumn{2}{|c|}{$\mathbf{k}_{1995}\left(\right.$ day $\left.^{-1}\right)$} & \multicolumn{2}{|c|}{$\mathbf{k}_{2015}\left(\right.$ day $\left.^{-1}\right)$} & \multicolumn{2}{|c|}{$\Delta k\left(\right.$ year $\left.^{-1}\right)$} \\
\hline & & & & & $0.496 \pm$ & 0.039 & & & $-0.4107 \pm$ & \pm 0.0235 & $-0.8554=$ & \pm 0.0236 & $0.0740=$ & \pm 0.0014 & & & & & & & & \\
\hline Chlordanes & vapor & $10 / 30 / 2004$ & 7970 & 0.271 & $0.519 \pm$ & 0.036 & $-1.60 \mathrm{E}-04 \pm$ & $9.35 \mathrm{E}-06$ & & & & & & & $6.25 \mathrm{E}-09 \pm$ & $\pm 4.09 \mathrm{E}-09$ & $-2.05 E-04=$ & $\pm 3.08 \mathrm{E}-05$ & $-1.14 \mathrm{E}-04 \pm$ & $\pm 3.56 \mathrm{E}-05$ & -0.0333 & \pm 0.0172 \\
\hline DTs & vapor & $10 / 4 / 2005$ & 33300 & 0.553 & $0.244 \pm$ & 0.039 & & & $-0.3656 \pm$ & \pm 0.0236 & $-0.9472=$ & \pm 0.0237 & $0.0676=$ & \pm 0.0014 & & & & & & & & \\
\hline DTs & vapor & $6 / 17 / 2004$ & 8250 & 0.358 & $0.273 \pm$ & 0.032 & $-1.74 \mathrm{E}-04 \pm$ & $8.16 \mathrm{E}-06$ & & & & & & & $6.78 \mathrm{E}-09 \pm$ & $\pm 3.46 \mathrm{E}-09$ & $-2.21 \mathrm{E}-04=$ & $\pm 2.53 \mathrm{E}-05$ & $-1.22 \mathrm{E}-04 \pm$ & $\pm 3.11 \mathrm{E}-05$ & -0.0362 & \pm 0.0146 \\
\hline ndosulfans & vapor & $6 / 1 / 20062$ & 29790 & 0.544 & $1.124 \pm$ & 0.057 & & & $-0.7303 \pm$ & \pm 0.0336 & $-1.7715=$ & \pm 0.0341 & $0.0387=$ & \pm 0.0020 & & & & & & & & \\
\hline indosulfans & vapor & $11 / 2 / 2005$ & 7230 & 0.379 & $1.422 \pm$ & 0.048 & $-2.64 \mathrm{E}-04 \pm$ & $1.36 \mathrm{E}-05$ & & & & & & & $-4.97 E-08 \pm$ & $51 \mathrm{E}-09$ & $1.30 \mathrm{E}-04=$ & $\pm 5.33 \mathrm{E}-05$ & $-5.96 \mathrm{E}-04 \pm$ & $\pm 5.19 \mathrm{E}-05$ & 0.2652 & \pm 0.0272 \\
\hline ICH, alpha & vapor & $9 / 11 / 20053$ & 32950 & 0.102 & $3.217 \pm$ & 0.049 & & & $-0.2567 \pm$ & \pm 0.0291 & $-0.3753=$ & \pm 0.0294 & -0.0209 & \pm 0.0018 & & & & & & & & \\
\hline CH, alpha & vapor & 6/13/2004 & 8180 & 0.904 & $3.267 \pm$ & 0.020 & $-4.41 \mathrm{E}-04 \pm$ & $5.08 \mathrm{E}-06$ & & & & & & & $2.45 \mathrm{E}-08 \pm$ & $\pm 2.15 \mathrm{E}-09$ & $-6.10 \mathrm{E}-04$ & $\pm 1.57 \mathrm{E}-05$ & $-2.53 \mathrm{E}-04 \pm$ & $\pm 1.94 \mathrm{E}-05$ & -0.1306 & \pm 0.0091 \\
\hline $\mathrm{CH}$, gamma & vapor & $7 / 3 / 20053$ & 31780 & 0.198 & $1.103 \pm$ & 0.054 & & & $-0.1250 \pm$ & \pm 0.0322 & $-0.8577=$ & \pm 0.0328 & 0.0168 & \pm 0.0020 & & & & & & & & \\
\hline $\mathrm{HCH}$, gamma & vapor & $6 / 21 / 2004$ & 8120 & 0.823 & $1.279 \pm$ & 0.028 & $-4.39 \mathrm{E}-04 \pm$ & 7.16E-06 & & & & & & & $-9.96 E-10 \pm$ & $\pm 3.02 \mathrm{E}-09$ & $-4.32 \mathrm{E}-04=$ & $\pm 2.21 \mathrm{E}-05$ & -4.47 & $\pm 2.72 \mathrm{E}-05$ & 0.0053 & $=0.0128$ \\
\hline AH, phen & vapor & $10 / 19 / 2005$ & 33220 & 0.752 & $4.134 \pm$ & 0.036 & & & $-0.2076 \pm$ & \pm 0.0219 & $-0.3829=$ & \pm 0.0220 & 0.1300 & \pm 0.0013 & & & & & & & & \\
\hline $\mathrm{AH}$, phen & vapor & 7/9/2004 & 8190 & 0.133 & $4.116 \pm$ & 0.031 & $-8.29 \mathrm{E}-05 \pm$ & $7.72 \mathrm{E}-06$ & & & & & & & $1.03 \mathrm{E}-08 \pm$ & $\pm 3.28 \mathrm{E}-09$ & $-1.54 \mathrm{E}-04=$ & $\pm 2.41 \mathrm{E}-05$ & $-4.15 \mathrm{E}-06 \pm$ & $\pm 2.94 \mathrm{E}-05$ & -0.0549 & \pm 0.0139 \\
\hline $\mathrm{AH}$, total & vapor & $10 / 21 / 2005$ & 33170 & 0.748 & $5.056 \pm$ & 0.035 & & & $-0.2311 \pm$ & \pm 0.0210 & $-0.3514=$ & \pm 0.0212 & 0.1237 & \pm 0.0013 & & & & & & & & \\
\hline $\mathrm{AH}$, total & vapor & $7 / 19 / 2004$ & 8160 & 0.156 & $5.056 \pm$ & 0.029 & $-8.74 \mathrm{E}-05 \pm$ & $7.26 \mathrm{E}-06$ & & & & & & & $7.11 \mathrm{E}-09 \pm$ & $\pm 3.09 \mathrm{E}-09$ & -1.37 & \pm 2.27 & -3.3 & $\pm 2.76 \mathrm{E}-05$ & -0.0379 & \pm 0.0131 \\
\hline CB, suite & vapor & $10 / 2 / 2005$ & 33090 & 0.715 & $2.709 \pm$ & 0.030 & & & $-0.3037 \pm$ & \pm 0.0181 & $-0.7419=$ & \pm 0.0182 & 0.0869 & & & & & & & & & \\
\hline CB, suit & vapor & i/2004 & 8240 & 0.375 & $2.816 \pm$ & 0.024 & $-1.31 \mathrm{E}-04 \pm$ & $5.97 \mathrm{E}-06$ & & & & & & & $-7.89 E-09 \pm$ & $\pm 2.54 \mathrm{E}-09$ & $-7.62 E-05=$ & \pm 1.85 & $-1.92 \mathrm{E}-04 \pm$ & $\pm 2.29 \mathrm{E}-05$ & 0.0421 & \pm 0.0108 \\
\hline CB-018 & vapor & $9 / 28 / 20$ & 33050 & 0.613 & $0.271 \pm$ & 0.034 & & & $-0.2216 \pm$ & \pm 0.0206 & $-0.6383=$ & \pm 0.0208 & 0.0803 & \pm 0.0013 & & & & & & & & \\
\hline CB-018 & vapor & $6 / 1 / 2004$ & 8260 & 0.505 & $0.412 \pm$ & 0.025 & 1.79E-04 \pm & $6.25 \mathrm{E}-06$ & & & & & & & $-8.92 E-09 \pm$ & $\pm 2.65 \mathrm{E}-09$ & $2.41 \mathrm{E}-04$ & \pm 1.93 & $1.10 \mathrm{E}-04 \pm$ & $\pm 2.40 \mathrm{E}-05$ & 0.0476 & \pm 0.0112 \\
\hline CB-052 & vapor & $10 / 4 / 2005$ & 33080 & 0.725 & $-0.144 \pm$ & 0.031 & & & $-0.3280 \pm$ & \pm 0.0183 & $-0.8102=$ & \pm 0.0185 & 0.0890 & \pm 0.0011 & & & & & & & & \\
\hline CB-052 & vapor & $6 / 1 / 2004$ & 8270 & 0.417 & $0.005 \pm$ & 0.023 & $-1.35 \mathrm{E}-04 \pm$ & $5.76 \mathrm{E}-06$ & & & & & & & $-1.39 E-08 \pm$ & $\pm 2.45 \mathrm{E}-09$ & $-3.97 E-05=$ & $\pm 1.78 \mathrm{E}-05$ & $-2.43 \mathrm{E}-04 \pm$ & $\pm 2.21 \mathrm{E}-05$ & 0.0741 & \pm 0.0104 \\
\hline B-1 & vapor & 10/6/ & 33080 & 0.728 & $-0.730 \pm$ & 0.033 & & & $-0.3703 \pm$ & \pm 0.0194 & $-0.8754=$ & \pm 0.0196 & 0.0946 & \pm 0.0012 & & & & & & & & \\
\hline CB-101 & vapor & 6/10/2004 & 8220 & 0.399 & $-0.612 \pm$ & 0.024 & $-1.38 \mathrm{E}-04 \pm$ & $6.01 \mathrm{E}-06$ & & & & & & & $-8.91 \mathrm{E}-09 \pm$ & $\pm 2.56 \mathrm{E}-09$ & $-7.65 E-05=$ & $\pm 1.87 \mathrm{E}-05$ & $-2.07 E-04 \pm$ & $\pm 2.31 \mathrm{E}-05$ & 0.0475 & \pm 0.0108 \\
\hline${ }^{*}$ & part. & & 8180 & 0.184 & $-2.365 \pm$ & 0.465 & & & $0.1516 \pm$ & \pm 0.0437 & $0.4881=$ & \pm 0.0445 & 0.0823 & & & & & & & & & \\
\hline lordan & irt. & & 5810 & 0.544 & $-2.478 \pm$ & 0.035 & $-2.96 \mathrm{E}-04 \pm$ & $1.17 \mathrm{E}-05$ & & & & & & & $4.31 \mathrm{E}-08 \pm$ & $\pm 6.08 \mathrm{E}-09$ & $-6.75 \mathrm{E}-04$ & \pm 5.4 & $-4.49 \mathrm{E}-05 \pm$ & $\pm 4.30 \mathrm{E}-05$ & -0.2301 & \pm 0.0254 \\
\hline DDTs* & rt. & $8 / 10 / 2006$ & 6800 & 0.148 & $-6.962 \pm$ & 0.703 & & & $0416 \pm$ & \pm 0.0655 & 0.1903 & \pm 0.0679 & 0.1769 & \pm 0.0169 & & & & & & & & \\
\hline DDTs* & part. & $3 / 26 / 2006$ & 5060 & 0.526 & $-7.124 \pm$ & 0.053 & $-4.49 \mathrm{E}-04 \pm$ & $1.90 \mathrm{E}-05$ & & & & & & & $5.31 \mathrm{E}-08 \pm$ & $\pm 9.16 \mathrm{E}-09$ & $-8.84 \mathrm{E}-04=$ & $\pm 7.75 \mathrm{E}-05$ & $-1.09 \mathrm{E}-04 \pm$ & $\pm 7.03 \mathrm{E}-05$ & -0.2831 & \pm 0.0382 \\
\hline indosulf & part. & $3 / 17 / 2007$ & 8100 & 0.049 & $3.510 \pm$ & 0.519 & & & $0.0651 \pm$ & \pm 0.0491 & $-0.2375=$ & \pm 0.0499 & -0.0522 & \pm 0.0125 & & & & & & & & \\
\hline ns* & part. & 11/17/2006 & 5750 & 0.560 & $3.833 \pm$ & 0.038 & $-3.18 \mathrm{E}-04 \pm$ & $1.29 \mathrm{E}-05$ & & & & & & & $-6.83 E-08 \pm$ & $5 E-09$ & $2.75 \mathrm{E}-04$ & \pm 5.9 & $-7.23 \mathrm{E}-04 \pm$ & $\pm 4.78 \mathrm{E}-05$ & 0.3644 & \pm 0.0278 \\
\hline & & $15 / 20072$ & 28360 & 0.733 & $0.892 \pm$ & 0.038 & & & $0.3599 \pm$ & \pm 0.0222 & 0.3847 & \pm 0.0224 & 0.1143 & \pm 0.0013 & & & & & & & & \\
\hline PAH, phen & part. & 11/9/2006 & 6630 & 0.250 & $0.804 \pm$ & 0.028 & $-1.23 \mathrm{E}-04 \pm$ & $8.77 \mathrm{E}-06$ & & & & & & & $2.22 \mathrm{E}-08 \pm$ & $\pm 4.53 \mathrm{E}-09$ & $-3.15 \mathrm{E}-04=$ & $\pm 4.02 \mathrm{E}-05$ & $9.59 \mathrm{E}-06 \pm$ & $\pm 3.26 \mathrm{E}-05$ & -0.1185 & \pm 0.0189 \\
\hline $\mathrm{AH}$ & part. & $4 / 5 / 2007$ & $2940 \mathrm{C}$ & 0.727 & $2.763 \pm$ & 0.045 & & & $0.3005 \pm$ & \pm 0.0264 & 0.5503 & \pm 0.0265 & 0.1350 & \pm 0.0016 & & & & & & & & \\
\hline $\mathrm{AH}$, total & part. & $11 / 14 / 2006$ & 6630 & 0.173 & $2.670 \pm$ & 0.034 & $-1.13 E-04 \pm$ & $1.05 \mathrm{E}-05$ & & & & & & & $2.47 \mathrm{E}-08 \pm$ & \pm 5.41E-09 & $-3.27 E-04=$ & $\pm 4.80 \mathrm{E}-05$ & $3.43 \mathrm{E}-05 \pm$ & $\pm 3.89 \mathrm{E}-05$ & -0.1319 & \pm 0.0226 \\
\hline & & & & & & & & & & & & & & & & & & & & & & \\
\hline
\end{tabular}




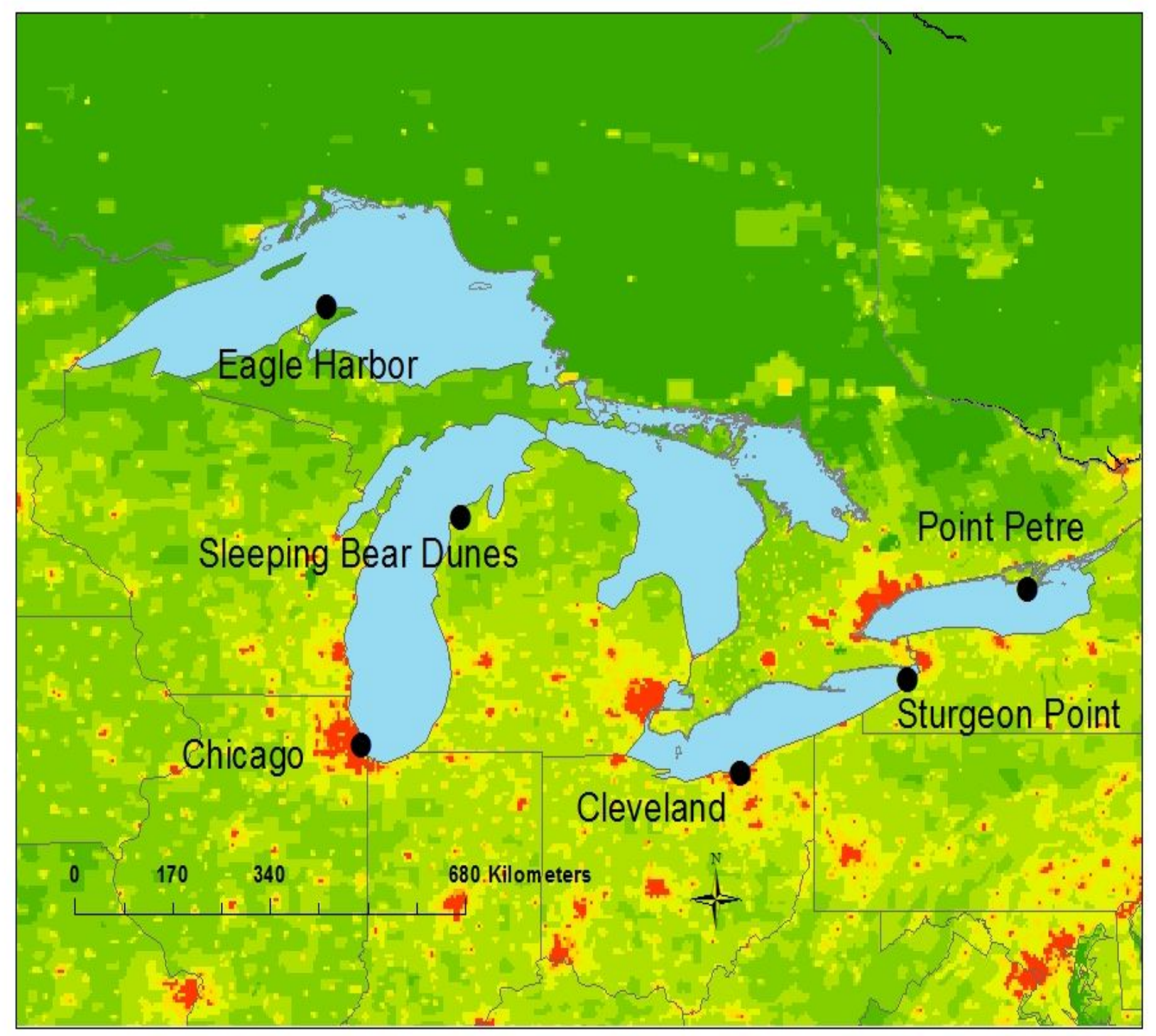

Figure S1. Map of the North American Great Lakes showing the six atmospheric sampling sites discussed in this paper. 